\title{
Mild Hypothermia Attenuates Changes in Respiratory System Mechanics and Modifies Cytokine Concentration in Bronchoalveolar Lavage Fluid During Low Lung Volume Ventilation
}

\author{
P. DOSTÁL ${ }^{1}$, M. ŠENKE ̌̌ÍK ${ }^{2}$, R. PAŘÍZKOVÁ ${ }^{1}$, D. BAREŠ ${ }^{1}$, P. ŽIVNÝ ${ }^{3}$, H. ŽIVNÁ \\ V. ČERNÝ ${ }^{1,5}$
}

${ }^{1}$ Department of Anesthesia and Intensive Care, Charles University Prague, Faculty of Medicine Hradec Králové, University Hospital Hradec Králové, Hradec Králové, Czech Republic,

${ }^{2}$ Department of Pediatrics, Charles University Prague, Faculty of Medicine Hradec Králové, University Hospital Hradec Králové, Hradec Králové, Czech Republic, ${ }^{3}$ Department of Clinical Biochemistry, Charles University Prague, Faculty of Medicine Hradec Králové, University Hospital Hradec Králové, Hradec Králové, Czech Republic, ${ }^{4}$ Radioisotope Laboratories and Vivarium, Charles University Prague, Faculty of Medicine Hradec Králové, University Hospital Hradec Králové, Hradec Králové, Czech Republic, ${ }^{5}$ Dalhousie University, Department of Anesthesia, Halifax, Canada

Received August 13, 2009

Accepted April 16, 2010

On-line June 9, 2010

\section{Summary}

Hypothermia was shown to attenuate ventilator-induced lung injury due to large tidal volumes. It is unclear if the protective effect of hypothermia is maintained under less injurious mechanical ventilation in animals without previous lung injury. Tracheostomized rats were randomly allocated to non-ventilated group (group C) or ventilated groups of normothermia (group $N$ ) and mild hypothermia (group $\mathrm{H}$ ). After two hours of mechanical ventilation with inspiratory fraction of oxygen 1.0, respiratory rate $60 \mathrm{~min}^{-1}$, tidal volume $10 \mathrm{ml} \mathrm{kg}^{-1}$, positive end-expiratory

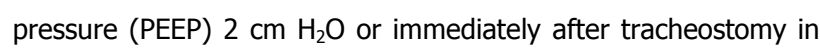
non-ventilated animals inspiratory pressures were recorded, rats were sacrificed, pressure-volume (PV) curve of respiratory system constructed, bronchoalveolar lavage (BAL) fluid and aortic blood samples obtained. Group N animals exhibited a higher rise in peak inspiratory pressures in comparison to group $\mathrm{H}$ animals. Shift of the PV curve to right, higher total protein and interleukin6 levels in BAL fluid were observed in normothermia animals in comparison with hypothermia animals and non-ventilated controls. Tumor necrosis factor- $\alpha$ was lower in the hypothermia group in comparison with normothermia and non-ventilated groups. Mild hypothermia attenuated changes in respiratory system mechanics and modified cytokine concentration in bronchoalveolar lavage fluid during low lung volume ventilation in animals without previous lung injury.

\section{Key words \\ Rats - Mechanical ventilation - Inflammation - Cytokines • Hypothermia \\ Corresponding author \\ P. Dostál, Department of Anesthesia and Intensive Care, University Hospital Hradec Králové, Sokolská 581, 500 05, Hradec Králové, Czech Republic. Fax: +420 49583 2022. E-mail: dostal@fnhk.cz}

\section{Introduction}

Hypothermia was shown to attenuate injury of the lung due to mechanical ventilation in several experimental studies that used large tidal volumes either alone or in combination with previous lung injury in both in vivo and ex vivo animal models of ventilator-induced lung injury (Lim et al. 2003, Suzuki et al. 2004, Hong et al. 2005). Although hypothermia could be considered as a 
means for lung rest in the acutely injured lung, because it may ensure lower frequency ventilation (Lim et al. 2003), the results of published studies (Lim et al. 2003, Suzuki et al. 2004, Akinci et al. 2005, Hong et al. 2005) suggest that temperature modulates activation of pulmonary resident cells and this may, at least partially, explain protective effects of hypothermia. It remains unclear if the protective effect of hypothermia is maintained under less injurious mechanical ventilation in animals without previous lung injury.

This study was designed to investigate whether mild hypothermia could attenuate the changes in respiratory system mechanics and cytokine concentration in bronchoalveolar lavage fluid during low PEEP, high inspiratory fraction of oxygen $\left(\mathrm{FiO}_{2}\right)$ and moderate tidal volume ventilation in animals without previous lung injury.

\section{Methods}

\section{Animal anesthesia and instrumentation}

The study protocol was approved by the Animal Care and Use Committee of the Faculty of Medicine in Hradec Králové, Charles University Prague.

Twenty-two male adult specific pathogen-free Sprague-Dawley rats (weighing 280 to $320 \mathrm{~g}$ ) were anesthetized using ether chamber, placed supine and Silastic (0.012-inch I.D, 0.025 inch O.D) cathethers were inserted in the left common carotid artery and in the right jugular vein. After awakening animals were returned to their cages and food and water was provided.

Twenty-four hours after instrumentation the rats were anesthetized by intraperitoneal administration of sodium pentobarbital (Nembutal, Abbott Laboratory, North Chicago, IL, USA) $50 \mathrm{mg} / \mathrm{kg}$ body weight. Additional pentobarbital was given freely as necessary. A tracheotomy was performed with a 14-gauge cannula (Vasocan Braunule, B.Braun, Germany) inserted to a depth of $1 \mathrm{~cm}$ and secured in place using three sutures. Mechanically ventilated animals were connected to a small animal ventilator (Inspiria ASV, Harvard Apparatus, MA) with an $\mathrm{FiO}_{2}$ of 1.0 , respiratory rate $60 / \mathrm{min}$, tidal volume (TV) $10 \mathrm{ml} / \mathrm{kg}$, inspiratory time $50 \%$ of respiratory cycle and PEEP $2 \mathrm{~cm} \mathrm{H}_{2} \mathrm{O}$.

Pancuronium (1 mg/kg; Organon, Oss, The Netherlands) was administered in mechanically ventilated animals intravenously after the depth of anesthesia was confirmed by the absence of response to paw compression. Depth of anesthesia was assessed every
$15 \mathrm{~min}$ by monitoring the hemodynamic response to paw clamp. Anesthesia was maintained with intravenously administered pentobarbital $(500 \mu \mathrm{g} / \mathrm{kg} / \mathrm{h})$, in case of response sodium pentobarbital $(2.5 \mathrm{mg})$ was given intravenously.

Muscle relaxation was maintained with pancuronium $(0.2 \mathrm{mg} / \mathrm{g} / \mathrm{h})$ administered by continuous infusion via the right jugular vein. Normal saline was also administered intravenously so that intravenous fluids totaled $4 \mathrm{ml} / \mathrm{h}$ including medications. The total volume of intravenous fluid and dosages of medications administered were recorded.

Airway pressures were monitored continuously (Gabarith PMSET 1DT-XX, Becton Dickenson, Singapore) and data recorded every $30 \mathrm{~min}$. A catheter in the left common carotid artery connected to a pressure transducer (Gabarith PMSET 1DT-XX, Becton Dickenson, Singapore) was used to continuously monitor blood pressure (Datex-Ohmeda $\mathrm{S} / 5$, Instrumentarium Corp., Helsinki, Finland). A central venous catheter placed in the right internal jugular vein was used to administer medications and fluids.

\section{Experimental groups and injurious ventilation}

After tracheostomy rats were randomly allocated to non-ventilated group $(\mathrm{C}, \mathrm{n}=6)$ or ventilated groups of normothermia $(\mathrm{N}, \mathrm{n}=8)$ and hypothermia $(\mathrm{H}, \mathrm{n}=8)$. In the normothermia group, body temperature was maintained using an electric blanket at $37 \pm 0.5{ }^{\circ} \mathrm{C}$ throughout the study, as measured $7 \mathrm{~cm}$ deep in the rectum. In the hypothermia group, rectal temperature was reduced to $33 \pm 0.5^{\circ} \mathrm{C}$ placing the bags with cold water $\left(7{ }^{\circ} \mathrm{C}\right)$ to the sides of the chest and proximal part of the abdomen. Cooling took up to 10 min depending on the size of the animal. The temperature of these animals was maintained in the target range until the end of the study. Fifteen minutes after the beginning of mechanical ventilation, baseline physiological values were recorded and mechanical ventilation using the previously described setting was maintained for two hours.

After two hours (immediately after tracheostomy in $\mathrm{C}$ group animals) heparin (400 IU/ $\mathrm{kg}$ ) and additional pentobarbital $(2.5 \mathrm{mg})$ were administered intravenously, abdomen was opened with a midline incision, the aorta visualized and animals were then killed by exsanguination from the abdominal aorta, the blood was collected into syringe. Exsanguination was performed on mechanical ventilation using unchanged ventilatory setting and inspiratory fraction of oxygen in both ventilated groups. 


\section{Physiological variables}

Systemic arterial pressure and peak airway pressure pressure were measured and recorded throughout the protocol and recorded every $30 \mathrm{~min}$ in both ventilated groups.

\section{Blood sampling}

At the end of the protocol an arterial blood sample was taken from the abdominal aorta and plasma was separated by centrifugation (centrifuge MPW-360, Mechanika Precyzyjna, Warszawa, Poland) at $10{ }^{\circ} \mathrm{C}$, $1500 \mathrm{~g}$ for $10 \mathrm{~min}$ and stored at $-70{ }^{\circ} \mathrm{C}$.

\section{Pressure-volume curve measurement}

Immediately after exsanguination the static pressure-volume (PV) curve was constructed. Animals were disconnected from mechanical ventilator for approximately $30 \mathrm{~s}$ and lungs were manually inflated using $20 \mathrm{ml}$ syringe with $2 \mathrm{ml}$ aliquots of air until total lung volume of $14 \mathrm{ml}$ or an airway pressure above $30 \mathrm{~mm} \mathrm{Hg}$ were reached. Airway pressures were recorded $5 \mathrm{~s}$ after every volume change.

\section{Bronchoalveolar lavage}

Immediately after pressure-volume curve measurements bronchoalveolar lavage (BAL) was performed. BAL was carried out by intratracheal instillation of four aliquots $(5 \mathrm{ml}$ each) of normal saline and returned fluid was collected by free drainage. Second aliquot of BAL fluid was used for laboratory analysis. Samples from the second of BAL fluid $(0.5 \mathrm{ml})$ were separated for the white blood cell count determination. The rest of BAL fluid samples were then centrifuged at $10{ }^{\circ} \mathrm{C}, 1000 \mathrm{~g}$ for $10 \mathrm{~min}$ and the supernatant was aspirated and stored at $-70{ }^{\circ} \mathrm{C}$ BAL fluid.

Determination of lung injury variables in BAL fluid and serum

The total protein concentrations were assayed as a measure of alveolar capillary permeability using the total protein colorimetric assay (Roche Diagnostics GmbH, Mannheim, Germany).

The white blood cell count was determined using a hemocytometer (Baxter Healthcare, McGaw Park, IL, USA).

Assays of tumor necrosis factor $\alpha(\mathrm{TNF}-\alpha)$ in the BAL supernatant and serum were performed with rat TNF-alfa UltraSensitive BioSource Immunoassay Kit (BioSource Europe S.A., Nivelles, Belgium).
IL-6 levels in the BAL supernatant and serum were measured using rat IL-6 BioSource immunoassay Kit (BioSource Europe S.A., Nivelles, Belgium).

\section{Statistical analysis}

Statistical analysis was performed with SigmaStat 3.1 software (Systat Software Inc., San Jose, CA, USA). For continuous variables the results are expressed as mean \pm S.D. for normally distributed data and as median /interquartile range/ where non-normally distributed. Data was analyzed by one-way ANOVA followed by Student-Newman-Keuls test or by one-way ANOVA on ranks followed by Dunn's test, by t-test or Mann-Whitney $\mathrm{U}$ test as appropriate. $\mathrm{P}<0.05$ value was accepted as statistically significant.

\section{Results}

\section{Physiological variables}

Arterial blood pressure values were stable during the study period and there were no significant differences between both ventilated groups.

Baseline peak airway pressures $(\mathrm{mm} \mathrm{Hg})$ did not differ between groups $(9.5 \pm 1.3$ in normothermia group vs $9.8 \pm 1.7$ in hypothermia group, $\mathrm{p}=0.798$ ). Peak airway pressures increased significantly during the study period in the normothermia group. The difference between groups reached significance after $2 \mathrm{~h}$ of mechanical ventilation (11.9 \pm 1.2 vs. $10.6 \pm 1.1, \mathrm{p}=0.049)$ (Fig. 1).

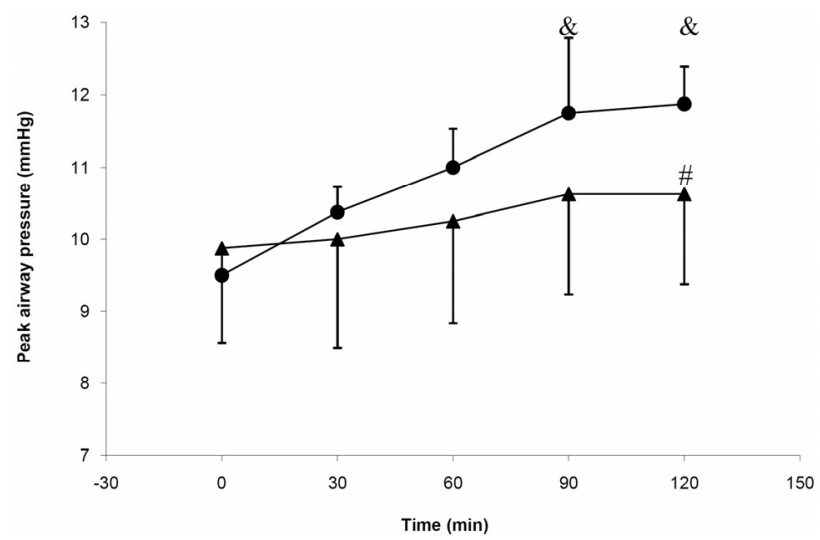

Fig. 1. Peak inspiratory pressures of the hypothermia (triangles) and normothermia (circles) groups. ${ }^{\#} \mathrm{p}<0.05$ compared with normothermia group, ${ }^{*} p<0.05$ compared with baseline value.

The PV curve of the normothermic group was significantly shifted to the right in comparison to the PV curve of the hypothermia group and the control group 


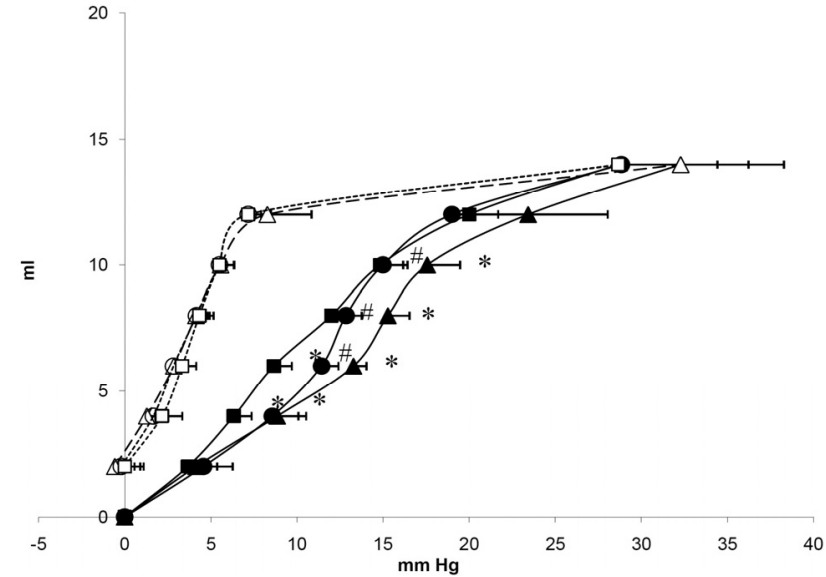

Fig. 2. Pressure-volume curve of the normothermia (triangles), hypothermia (circles) and non-ventilated (squares) groups. $* p<0.05$ compared with non-ventilated group, $p<0.05$ compared with normothermia group (empty symbols - deflation limb).

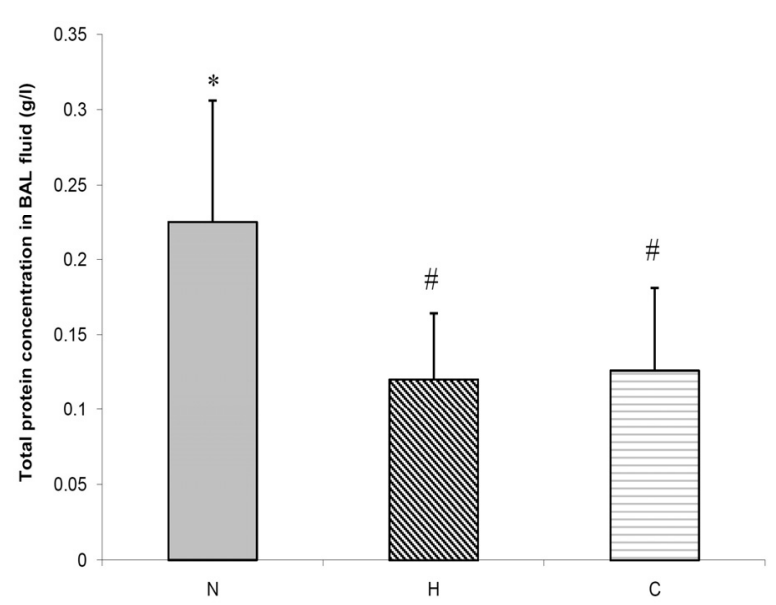

Fig. 3. The protein concentration in bronchoalveolar lavage (BAL) fluid in experimental groups $(\mathrm{N}$ - normothermia group; $\mathrm{H}-$ hypothermia group; $\mathrm{C}-$ non-ventilated group). $* p<0.05$ compared with non-ventilated group, $p<0.05$ compared with normothermia group.

(Fig. 2). The static airway pressures of the hypothermia group were lower than those of the normothermia group at volumes 6,8 , and $10 \mathrm{ml}$ above the end-expiratory volume (all $\mathrm{p}<0.05$ ). Both ventilated groups showed higher static pressures in comparison with the control group at low lung volumes (4 and $6 \mathrm{ml}$ above the endexpiratory volume, all $\mathrm{p}<0.05$ ).

\section{Lung injury variables in BAL fluid}

The harvested amount of BAL fluid was not different between groups. The protein concentration (g/l) in the BAL fluid of the normothermia group was higher than that of the hypothermia group and control group $(0.225 \pm 0.082$ vs. $0.120 \pm 0.044$ vs. $0.126 \pm 0.055$, respectively, $\mathrm{p}=0.009$ ) (Fig. 3).

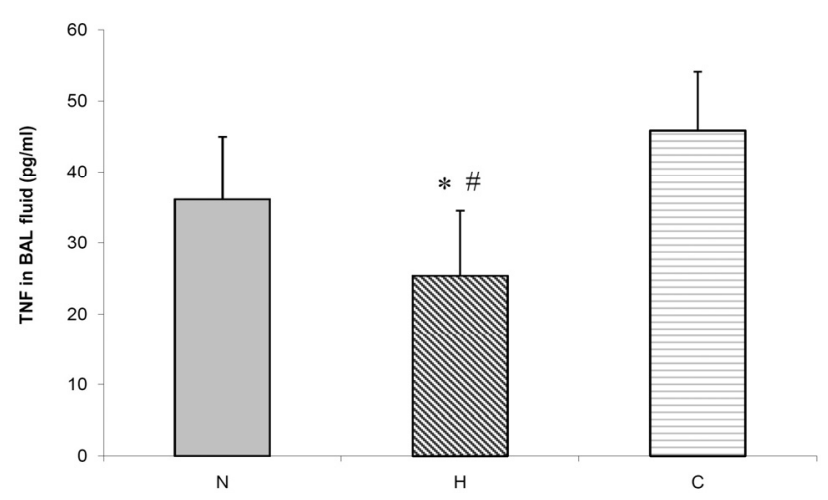

Fig. 4. Tumor necrosis factor a (TNF) level in bronchoalveolar lavage (BAL) fluid in experimental groups $(\mathrm{N}$ - normothermia group; $\mathrm{H}$ - hypothermia group; $\mathrm{C}$ - non-ventilated group). $* p<0.05$ compared with non-ventilated group, $p<0.05$ compared with normothermia group.

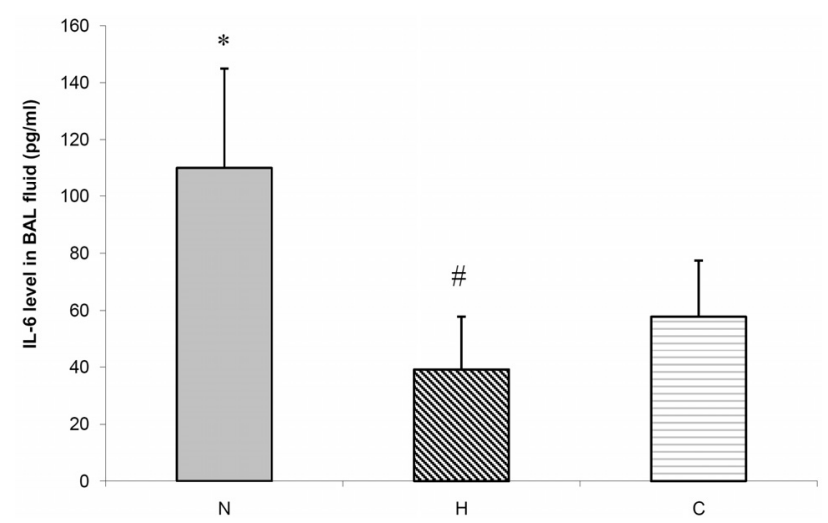

Fig. 5. Interleukin-6 (IL-6) level in bronchoalveolar lavage (BAL) fluid in experimental groups ( $\mathrm{N}$ - normothermia group; $\mathrm{H}-$ hypothermia group; $\mathrm{C}-$ non-ventilated group). $* \mathrm{p}<0.05$ compared with non-ventilated group, $p<0.05$ compared with normothermia group.

The WBC counts in the BAL fluid $\left(10^{4} / \mathrm{ml}\right)$ were not significantly different among the normothermia, hypothermia and control groups $(8.0 / 5.5 ; 15.5 / \mathrm{vs}$. $6.0 / 4.0 ; 8.0 /$ vs. $6.5 / 5.0 ; 12.0 /, \mathrm{p}=0.433)$.

The TNF level in the BAL fluid $(\mathrm{pg} / \mathrm{ml})$ was lower in the hypothermia group both in comparison with the normothermia group (25.4 \pm 9.1 vs. $36.1 \pm 8.9, \mathrm{p}=0.036)$ and the control group ( $25.4 \pm 9.1$ vs. $45.9 \pm 8.2, \mathrm{p}<0.001)$. There was no significant difference between the control and normothermia groups $(45.9 \pm 8.2$ vs. $36.1 \pm 8.9$, $\mathrm{p}=0.059$ ) (Fig. 4).

The IL-6 level in the BAL fluid (pg/ml) was higher in the normothermia group both in comparison with the hypothermia group $(110.1 \pm 34.9$ vs. $39.2 \pm 18.7$, $\mathrm{p}<0.001)$ and the control group $(110.1 \pm 34.9$ vs. $57.9 \pm 19.6, \mathrm{p}=0.001)$. There was no significant difference between the control and hypothermia groups $(57.9 \pm 19.6$ vs. $39.2 \pm 18.7, \mathrm{p}=0.168$ ) (Fig. 5). 


\section{Lung injury variables in serum}

Serum TNF $(\mathrm{pg} / \mathrm{ml})$ were similar in the normothermia, hypothermia and control groups ( $1.01 \pm 0.71$ vs. $1.28 \pm 1.11$ vs. $1.3 \pm 0.56, \mathrm{p}=0.720)$. Serum IL-6 was below the detection limit in all groups.

\section{Discussion}

Our main findings were that animals without previous lung injury ventilated at body temperature $33^{\circ} \mathrm{C}$ had lower changes in mechanical properties of respiratory system and lower levels of lung injury markers (cytokines and total protein concentrations) in BAL fluid after two hours of mechanical ventilation with moderate tidal volumes, low PEEP and high inspiratory fraction of oxygen.

Prolonged mechanical ventilation at low lung volume and zero end-expiratory pressure using physiological tidal volumes has been shown to induce histological evidence of peripheral airway injury and parenchymal inflammation with a concomitant increase in airway resistance, which persists after restoration of physiological end-expiratory lung volume (D'Angelo et al. 2002, 2004). In these studies, morphological and mechanical alterations have been attributed to shear stresses caused by cyclic opening and closing of peripheral airways with tidal ventilation at low lung volumes, possibly combined with increased surface tension due to surfactant depletion or inactivation (Robertson 1984). Recent study (D’Angelo et al. 2005) also showed that mechanical ventilation at low volume with physiological tidal volumes causes an increase in airway resistance in rabbits. In closed-chest animals, this increase was more pronounced and associated with an increase of lung elastance, likely due to interstitial edema and surfactant depletion or inactivation.

We used low PEEP, high $\mathrm{FiO}_{2}$ and moderate tidal volumes in this study to mimic the ventilatory setting sometimes utilized temporarily in patients with intracranial hypertension and low brain tissue oxygen tension. High concentration of oxygen is known to accelerate derecruitment in lung areas with low ventilation/perfusion ratio (Aboab et al. 2006). The shape of PV curves in both ventilated groups showed initial low compliance and lower inflection point that are considered markers of airway and/or alveolar collapse or closure (Martin-Lefevre et al. 2001). We therefore speculate that high inspiratory fraction of oxygen used in this study even in the presence of low PEEP increased the magnitude of lung derecruitment observed to occur in both ventilated groups and that mechanical forces applied in this model were probably similar to previously published low lung volume injury models.

Increased total protein concentration in BAL fluid is considered to be a marker of increased alveolar capillary permeability (Behnia et al. 1996, Koay et al. 2002). The lower protein content in the bronchoalveolar fluid of the hypothermia group suggests a protective effect of hypothermia. Similar results have been observed in previous studies on hypothermia (Lim et al. 2003, Hong et al. 2005).

Mechanical ventilation itself may increase TNF- $\alpha$ in the lung (Imai et al. 1999) and cytokines levels in BAL fluid correlated with degree of lung injury due to mechanical ventilation (Tremblay et al. 1997). Hypothermia also decreases activation of nuclear factor$\kappa \mathrm{B}$ in the lung, (Koay et al. 2002) and this has been suggested to be one of the possible protective mechanisms of hypothermia (Hong et al. 2005). Lower TNF- $\alpha$ BAL fluid concentration observed in the hypothermia group probably reflects this mechanism.

Neutrophils are known as important inflammatory cells involved in the development of ventilator-induced lung injury (Kawano et al. 1987, Markos et al. 1993). In various settings, hypothermia has been shown to inhibit tissue accumulation of neutrophils (Toyoda et al. 1996, Whalen et al. 1997). In comparison with previous studies that used more injurious mechanical ventilation (Lim et al. 2003, Hong et al. 2005) we were not able to show a significant difference in number of WBCs in BAL fluid, although there was a non-significant trend towards higher values in the normothermia group.

Gradual rise of peak inspiratory pressure observed in normothermia group reflected deterioration of effective compliance of respiratory system. For technical reasons we did not measure inspiratory and expiratory pressures under static conditions and therefore we are not able to differentiate between increased resistance, decreased static compliance or increased intrinsic PEEP. PV curves of both mechanically ventilated groups showed the lower and upper inflection points. The inspiratory limb of the PV curve of the normothermia group was shifted to the right indicating more profound loss of aerated lung tissue and/or airway closure.

We observed similar course of PV curve during deflation in all groups. This could be explained by the 
gentle nature of insult using moderate tidal volumes and by the reversibility of loss of aerated lung tissue and/or airway closure after full recruitment of the lung during the PV curve measurement. Taking into account lower total protein, IL- 6 and TNF- $\alpha$ levels in BAL fluid of the hypothermia group we attribute observed changes in mechanical properties of respiratory system to less significant inflammatory response to applied insult.

Mechanical ventilation using the same setting of ventilator had to lead to lower arterial carbon dioxide tension in blood and tissues under hypothermia. The attenuated changes in mechanical properties and cytokine levels in BAL fluid observed in the hypothermia group appear to be in opposition to certain recent reports in which respiratory alkalosis was shown to be lungdeleterious while respiratory acidosis was lung-protective (Shibata et al. 1998, Laffey et al. 2000, 2004, Sinclair et al. 2002). It seems that the potential adverse effect of respiratory alkalosis is less important than the protective effect of hypothermia. Mild hypothermia may protect the lung by several mechanisms. Decreased metabolic rate may enable so called "lung rest" decreasing ventilation requirements (Hong et al. 2005). Since the same setting of ventilator was used in both groups, this mechanism does not explain the observed differences. Hypothermia may induce changes in cardiac output, lung perfusion and lung microcirculation; these parameters beside systemic blood pressure were not measured. Decreased lung perfusion was described to modify the extent of lung injury in ex vivo perfused lung models of ventilatorinduced lung injury (Broccard et al. 1998). However, observations published by Suzuki et al. (2004) suggest that hypothermia protects the lung by mechanisms that are unrelated to lung perfusion. Our data are in concordance with previously published results (Sarcia et al. 2003, Lim et al. 2004, Suzuki et al. 2004) suggesting that temperature-dependent modulation of inflammatory response activation seems to be at least partially responsible for protective effect of hypothermia in setting of ventilator-induced lung injury.

Our study has several methodological limitations. Due to using high inspiratory fraction of oxygen we are not able to differentiate between the influence of hypothermia on the effects of hyperoxia from the influence of hypothermia on the effects of mechanical ventilation. However, a recent experimental study showed that moderate hyperoxia does not increase lung injury in the absence of high lung volume injury (Sinclair et al. 2004). Even considering this information it remains unclear whether the inspiratory fraction of oxygen affected the results of our study.

For technical reasons we did not use classical markers of lung injury like histological score or lung wet/dry ratio for comparison between groups. Even considering absence of direct markers of lung injury in our study, we believe that used estimation of mechanical properties of respiratory system and used biochemical markers measured in BAL fluid are acceptable indirect indicators of adverse effect of mechanical ventilation on lung structure.

In theory, the differences in BAL fluid concentrations may be due to different dilution of bronchoalveolar fluid and different distribution of BAL fluid during bronchoalveolar lavage. The amount of harvested BAL fluid was not different between groups. BAL was performed after PV curve (e.g. recruitment maneuver) to sample most of the lung and the deflation limb of PV curve suggests similar lung volume at the end of procedure. Observed differences in levels of used markers between groups can not be simply explained by uneven dilution of bronchoalveolar fluid during BAL.

Last but not least the length of experiment was also limited and therefore we cannot exclude only temporary effect of hypothermia. This is a clear limitation, which could have been overcome by modifying the protocol only. Interspecies differences may also reduce translation of our results to the clinical setting.

In conclusions, mild hypothermia attenuated changes in respiratory system mechanics and modified cytokine concentration in bronchoalveolar lavage fluid during low lung volume ventilation.

\section{Conflict of Interest}

The authors have no competing interests and financial relationships that influence the presentation of data in this article.

\section{Acknowledgements}

This study was supported Research project MZO 00179906. 


\section{References}

ABOAB J, JONSON B, KOUATCHET A, TAILLE S, NIKLASON L, BROCHARD L: Effect of inspired oxygen fraction on alveolar derecruitment in acute respiratory distress syndrome. Intensive Care Med 32: 1979-1986, 2006.

AKINCI OI, CELIK M, MUTLU GM, MARTINO M, TUGRIK S, OZVÁN PE, YILMAZBAYHAN D, YELDANDI AV, TURKOZ KH, KIREN B, TELCI L, CAKAR N: Effect of body temperature on ventilator-induced lung injury. J Crit Care 20: 66-73, 2005.

BEHNIA R, MOLTENI A, WATERS CM, PANOS RJ, WARD WF, SCHNAPER HW, TS'AO CH: Early markers of ventilator-induced lung injury in rats. Ann Clin Lab Sci 26: 437-450, 1996.

BROCCARD AF, HOTCHKISS JR, KUWAYAMA N, OLSON DA, JAMAL S, WANGENSTEEN DO, MARINI JJ: Consequences of vascular flow on lung injury induced by mechanical ventilation. Am J Respir Crit Care Med 157: 1935-1942, 1998.

D'ANGELO E, PECCHIARI M, BARAGgIA P, SAETTA M, BALESTRO E, MILIC-EMILI J: Low-volume ventilation causes peripheral airway injury and increased airway resistance in normal rabbits. $J$ Appl Physiol 92: 949-956, 2002.

D'ANGELO E, PECCHIARI M, SAETTA M, BALESTRO E, MILIC-EMILI J: Dependence of lung imjury on inflation rate during low-volume ventilation in normal open-chest rabbits. $J$ Appl Physiol 97: 260-268, 2004.

D'ANGElO E, PECCHIARI M, Della VALle P, KOUTSOUKOU A, MILIC-EMILI J: Effects of mechanical ventilation at low lung volume on respiratory mechanics and nitric oxide exhalation in normal rabbits. $J$ Appl Physiol 99: 433-444, 2005.

HONG SB, KOH Y, LEE IC, KIM MJ, KIM WS, KIM DS, KIM WD, LIM CM: Induced hypothermia as a new approach to lung rest for the acutely injured lung. Crit Care Med 33: 2049-2055, 2005.

IMAI Y, KAWANO T, IWAMOTO S, NAKAGAWA S, TAKATA M, MIYASAKA K: Intratracheal anti-tumor necrosis factor-alpha antibody attenuates ventilator-induced lung injury in rabbits. $J$ Appl Physiol 87: 510-515, 1999.

KAWANO T, MORI S, CYBULSKY M, BURGER R, BALLIN A, CUTZ E, BRYAN AC: Effect of granulocyte depletion in a ventilated surfactant-depleted lung. $J$ Appl Physiol 62: 27-33, 1987.

KOAY MA, GAO X, WASHINGTON MK, PARMAN KS, SADIKOT RT, BLACKWELL TS, CHRISTMAN JW: Macrophages are necessary for maximal nuclear factor-kappa B activation in response to endotoxin. $A m J$ Respir Cell Mol Biol 26: 572-578, 2002.

LAFFEY JG, ENGELBERTS D, KAVANAGH BP: Injurious effects of hypocapnic alkalosis in the isolated lung. Am J Respir Crit Care Med 162: 399-405, 2000.

LAFFEY JG, O'CROININ D, MCLOUGHLIN P, KAVANAGH BP: Permissive hypercapnia - role in protective lung ventilatory strategies. Intensive Care Med 30: 347-356, 2004.

LIM CM, HONG SB, KOH Y, LEE SD, KIM WS, KIM DS, KIM WD: Hypothermia attenuates vascular manifestations of ventilator-induced lung injury in rats. Lung 181: 23-34, 2003.

LIM CM, KIM EK, KOH Y, KIM WS, KIM DS, KIM WD: Hypothermia inhibits cytokine release of alveolar macrophage and activation of nuclear factor kappaB in endotoxemic lung. Intensive Care Med 30: 1638-1644, 2004.

MARKOS J, DOERSCHUK CM, ENGLISH D, WIGGS BR, HOGG JC: Effect of positive end-expiratory pressure on leukocyte transit in rabbit lungs. $J$ Appl Physiol 74: 2627-2633, 1993.

MARTIN-LEFEVRE L, RICARD JD, ROUPIE E, DREYFUSS D, SAUMON G: Significance of the changes in the respiratory system pressure-volume curve during acute lung injury in rats. Am J Respir Crit Care Med 164: 627-632, 2001.

ROBERTSON B: Lung surfactant. In: Pulmonary Surfactant. B ROBERTSON, L VAN GOULDE, J BATENBURG (eds), Elsevier, Amsterdam, 1984, pp 132-147.

SARCIA PJ, SCUMPIA PO, MOLDAWER LL, DEMARCO VG, SKIMMING JW: Hypothermia induces interleukin10 and attenuates injury in the lungs of endotoxemic rats. Shock 20: 41-45, 2003. 
SHIBATA K, CREGG N, ENGELBERTS D, TAKEUCHI A, FEDORKO L, KAVANAGH BP: Hypercapnic acidosis may attenuate acute lung injury by inhibition of endogenous xanthine oxidase. Am J Respir Crit Care Med 158: $1578-1584,1998$.

SINCLAIR SE, ALTEMEIER WA, MATUTE-BELLO G, CHI EY: Augmented lung injury due to interaction between hyperoxia and mechanical ventilation. Crit Care Med 32: 2496-2501, 2004.

SINCLAIR SE, KREGENOW DA, LAMM WJ, STARR IR, CHI EY, HLASTALA MP: Hypercapnic acidosis is protective in an in vivo model of ventilator-induced lung injury. Am J Respir Crit Care Med 166: 403-408, 2002.

SUZUKI S, HOTCHKISS JR, TAKAHASHI T, OLSON D, ADAMS AB, MARINI JJ: Effect of core body temperature on ventilator-induced lung injury. Crit Care Med 32: 144-149, 2004.

TOYODA T, SUZUKI S, KASSELL NF, LEE KS: Intraischemic hypothermia attenuates neutrophil infiltration in the rat neocortex after focal ischemia-reperfusion injury. Neurosurgery 39: 1200-1205, 1996.

TREMBLAY L, VALENZA F, RIBEIRO SP, LI J, SLUTSKY AS: Injurious ventilatory strategies increase cytokines and c-fos m-RNA expression in an isolated rat lung model. J Clin Invest 99: 944-952, 1997.

WHALEN MJ, CARLOS TM, CLARK RS, MARION DW, DEKOSKY MS, HEINEMAN S, SCHIDING JK, MEMARZADEH F, DIXON CE, KOCHANEK PM: The relationship between brain temperature and neutrophil accumulation after traumatic brain injury in rats. Acta Neurochir 70 (Suppl): 260-261, 1997. 